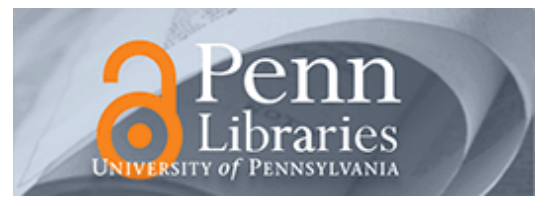

University of Pennsylvania

ScholarlyCommons

$1-5-2010$

\title{
Single-scattering Optical Tomography: Simultaneous Reconstruction of Scattering and Absorption
}

\author{
Lucia Florescu \\ University of Pennsylvania \\ Vadim A. Markel \\ University of Pennsylvania, vmarkel@mail.med.upenn.edu \\ John C. Schotland \\ University of Pennsylvania, schotland@seas.upenn.edu
}

Follow this and additional works at: https://repository.upenn.edu/be_papers

Part of the Biomedical Engineering and Bioengineering Commons

\section{Recommended Citation}

Florescu, L., Markel, V. A., \& Schotland, J. C. (2010). Single-scattering Optical Tomography: Simultaneous Reconstruction of Scattering and Absorption. Retrieved from https://repository.upenn.edu/be_papers/160

Suggested Citation:

Florescu, L., V. Markel, and J.C. Schotland. (2010). "Single-scattering optical tomography: Simultaneous reconstruction of scattering and absorption." Physical Review E. 81, 016602.

(C) 2010 The American Physical Society http://dx.doi.org/10.1103/PhysRevE.81.016602.

This paper is posted at ScholarlyCommons. https://repository.upenn.edu/be_papers/160

For more information, please contact repository@pobox.upenn.edu. 


\title{
Single-scattering Optical Tomography: Simultaneous Reconstruction of Scattering and Absorption
}

\author{
Abstract \\ We report theory and numerical simulations that demonstrate the feasibility of simultaneous \\ reconstruction of the three-dimensional scattering and absorption coefficients of a mesoscopic system \\ using angularly resolved measurements of scattered light. Image reconstruction is based on the inversion \\ of a generalized (broken ray) Radon transform relating the scattering and absorption coefficients of the \\ medium to angularly resolved intensity measurements. Although the single-scattering approximation to \\ the radiative transport equation (RTE) is used to devise the image reconstruction method, there is no \\ assumption that only singly scattered light is measured. That is, no physical mechanism for separating \\ single-scattered photons from the rest of the multiplyscattered light (e.g., time gating) is employed in the \\ proposed experiments. Numerical examples of image reconstruction are obtained using samples of \\ optical depth of up to 3.2. The forward data are obtained from numerical solution of the RTE, accounting \\ for all orders of scattering.

\section{Disciplines} \\ Biomedical Engineering and Bioengineering | Engineering

\section{Comments} \\ Suggested Citation: \\ Florescu, L., V. Markel, and J.C. Schotland. (2010). "Single-scattering optical tomography: Simultaneous \\ reconstruction of scattering and absorption." Physical Review E. 81, 016602. \\ (c) 2010 The American Physical Society http://dx.doi.org/10.1103/PhysRevE.81.016602.
}




\title{
Single-scattering optical tomography: Simultaneous reconstruction of scattering and absorption
}

\author{
Lucia Florescu \\ Department of Bioengineering, University of Pennsylvania, Philadelphia, Pennsylvania 19104, USA \\ Vadim A. Markel \\ Departments of Radiology and Bioengineering, University of Pennsylvania, Philadelphia, Pennsylvania 19104, USA \\ John C. Schotland \\ Department of Bioengineering and Graduate Group in Applied Mathematics and Computational Science, University of Pennsylvania, \\ Philadelphia, Pennsylvania 19104, USA \\ (Received 26 June 2009; published 5 January 2010)
}

\begin{abstract}
We report theory and numerical simulations that demonstrate the feasibility of simultaneous reconstruction of the three-dimensional scattering and absorption coefficients of a mesoscopic system using angularly resolved measurements of scattered light. Image reconstruction is based on the inversion of a generalized (broken ray) Radon transform relating the scattering and absorption coefficients of the medium to angularly resolved intensity measurements. Although the single-scattering approximation to the radiative transport equation (RTE) is used to devise the image reconstruction method, there is no assumption that only singly scattered light is measured. That is, no physical mechanism for separating single-scattered photons from the rest of the multiplyscattered light (e.g., time gating) is employed in the proposed experiments. Numerical examples of image reconstruction are obtained using samples of optical depth of up to 3.2. The forward data are obtained from numerical solution of the RTE, accounting for all orders of scattering.
\end{abstract}

DOI: 10.1103/PhysRevE.81.016602

PACS number(s): 42.30.Wb

\section{INTRODUCTION}

There is considerable interest in the development of techniques for three-dimensional optical imaging of biological systems. One application that is particularly appealing is the study of mesoscopic systems such as engineered tissues and model organisms, including Drosophila and Zebra fish [1]. For such systems, the photon transport mean free path is on the same order as the system size [2]. As a consequence, imaging modalities that assume either ballistic [3-6] or diffuse [7] propagation of light are not suitable. Thus, optical imaging techniques that bridge the gap between microscopic and macroscopic scales are of current importance [1].

In Ref. [8], we have proposed an imaging technique that makes use of angularly selective intensity measurements to reconstruct the total attenuation coefficient of an inhomogeneous medium, assuming that the measured light is predominantly single-scattered. This technique, which is referred to as single-scattering optical tomography (SSOT), takes as its starting point a generalization of the Radon transform in which the integral of the attenuation coefficient $\mu_{t}$ along a broken ray (which corresponds to the path of a singlescattered photon) is related to the measured intensity. It can be shown that this relationship may be inverted to recover the attenuation coefficient of the medium. We have found that SSOT produces high-quality images even in relatively thick samples, where the single-scattering approximation is expected to break down. It is important to emphasize that the experiments proposed in Ref. [8] do not rely on any physical mechanism for separating single-scattered photons from the remainder of the scattered light (such as time gating, for example) and are, therefore, relatively simple.

In this paper, we generalize our previous results by demonstrating that it is possible to separately recover the scatter- ing and absorption coefficients of the medium, $\mu_{a}$ and $\mu_{s}$, by making use of intensity measurements in two outgoing directions $\left(\mu_{t}=\mu_{a}+\mu_{s}\right)$. Thus, we show that the effects of scattering can be used to infer additional information about the medium. Previously, scattering in mesoscopic systems has been viewed as an obstruction to imaging. Thus, the effects of scattering are not taken into account and typically result in image degradation.

This paper is organized as follows. In Sec. II, we briefly review the theory of SSOT and then specialize to the case of normal incidence and two-angle detection which is further explored in detail. In Sec. III, we describe the numerical procedures we employ and in Sec. IV report our results. Finally, Sec. V contains concluding remarks.

\section{THEORY}

Consider a spatially nonuniform medium filling a slab of width $L$. Suppose that an incident beam of light of intensity $I_{0}$ enters the slab at the point $\mathbf{r}_{1}$ in the direction $\hat{\mathbf{s}}_{1}$. An angularly selective detector registers the ray exiting the slab through the opposite surface at the point $\mathbf{r}_{2}$ in the direction $\hat{\mathbf{s}}_{2}$ (as shown in Fig. 1). The intensity measured in such an experiment is denoted by $I_{s}\left(\mathbf{r}_{2}, \hat{\mathbf{s}}_{2} ; \mathbf{r}_{1}, \hat{\mathbf{s}}_{1}\right)$. Note that the backscattering measurement geometry can also be employed without any additional complications.

We assume that scattering is not very strong, that is $\mu_{s} L$ $=O(1)$, and that the single-scattering approximation to the radiative transport equation (RTE) can be adopted. Note, however, that in the numerical simulations reported below, the forward data are obtained by solving the RTE without any approximations, accounting for all orders of scattering. In the single-scattering approximation, the quantities $\mu_{t}$ and 


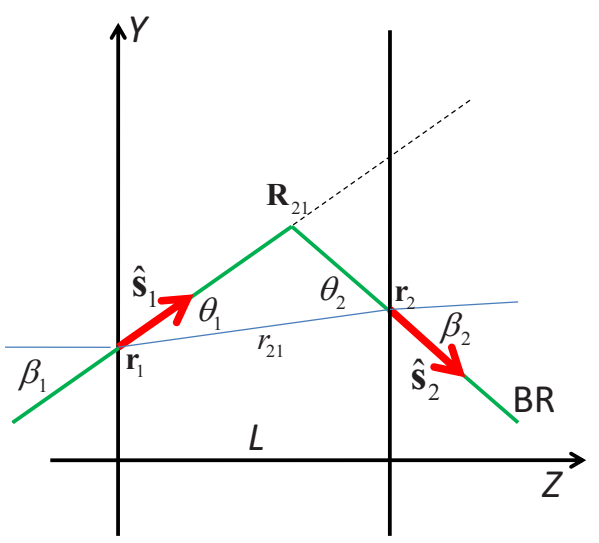

FIG. 1. (Color online) Illustrating the quantities used in Eq. (3) and elsewhere. "BR" denotes broken ray.

$\mu_{s}$ are related to the measured light intensity by [8]

$$
\begin{aligned}
I_{s}\left(\mathbf{r}_{1}, \hat{\mathbf{s}}_{1} ; \mathbf{r}_{2}, \hat{\mathbf{s}}_{2}\right)= & I_{0} \Theta\left(\pi-\theta_{1}-\theta_{2}\right) \delta\left(\left|\varphi_{\hat{\mathbf{s}}_{1}}-\varphi_{\hat{\mathbf{s}}_{2}}\right|-\pi\right) \\
& \times \frac{\mu_{s}\left(\mathbf{R}_{21}\right) A\left(\hat{\mathbf{s}}_{2}, \hat{\mathbf{s}}_{1}\right)}{r_{21} \sin \theta_{1} \sin \theta_{2}} \\
& \times \exp \left\{-\int_{\mathrm{BR}\left(\mathbf{r}_{2}, \hat{\mathbf{s}}_{2} ; \mathbf{r}_{1}, \hat{\mathbf{s}}_{1}\right)} \mu_{t}[\mathbf{r}(\ell)] d \ell\right\} .
\end{aligned}
$$

Here, $\Theta(x)$ is the unit step function, $A\left(\hat{\mathbf{s}}_{2}, \hat{\mathbf{s}}_{1}\right)$ is the phase function, $r_{21}=\left|\mathbf{r}_{2}-\mathbf{r}_{1}\right|$ is the distance between the source and the detector, the angles $\theta_{1}$ and $\theta_{2}$ are defined in Fig. 1 and the quantities $\varphi_{\hat{\mathbf{s}}_{1}}, \varphi_{\hat{\mathrm{s}}_{2}}$ are the azimuthal angles of vectors $\hat{\mathbf{s}}_{1}, \hat{\mathbf{s}}_{2}$ with respect to the $z$ axis. Note that the factor $\delta\left(\left|\varphi_{\hat{\mathbf{s}}_{1}}-\varphi_{\hat{\mathbf{s}}_{2}}\right|-\pi\right)$ is nonzero only when the two vectors $\hat{\mathbf{s}}_{1}, \hat{\mathbf{s}}_{2}$ are in the same plane. This particular case is illustrated in Fig. 1. The integral in Eq. (1) is evaluated along the broken ray (denoted "BR"), corresponding to the path of a singlescattered photon, and $\mathbf{R}_{21}$ is the turning point of this ray.

Equation (1) can be rewritten in the form

$$
\phi\left(\mathbf{r}_{2}, \hat{\mathbf{s}}_{2} ; \mathbf{r}_{1}, \hat{\mathbf{s}}_{1}\right)=\int_{\mathrm{BR}\left(\mathbf{r}_{2}, \hat{\mathbf{s}}_{2} ; \mathbf{r}_{1}, \hat{\mathbf{s}}_{1}\right)} \mu_{t}[\mathbf{r}(\ell)] d \ell-\ln \left[\frac{\mu_{s}\left(\mathbf{R}_{21}\right)}{\bar{\mu}_{s}}\right],
$$

where $\bar{\mu}_{s}$ is the reference (background) value of the scattering coefficient, and the data function $\phi\left(\mathbf{r}_{2}, \hat{\mathbf{s}}_{2} ; \mathbf{r}_{1}, \hat{\mathbf{s}}_{1}\right)$ is defined by

$$
\phi\left(\mathbf{r}_{2}, \hat{\mathbf{s}}_{2} ; \mathbf{r}_{1}, \hat{\mathbf{s}}_{1}\right)=-\ln \left[\frac{r_{21} \sin \theta_{1} \sin \theta_{2} \int I_{s}\left(\mathbf{r}_{2}, \hat{\mathbf{s}}_{2} ; \mathbf{r}_{1}, \hat{\mathbf{s}}_{1}\right) d \varphi_{\hat{\mathbf{s}}_{2}}}{I_{0} \bar{\mu}_{s} A\left(\hat{\mathbf{s}}_{2}, \hat{\mathbf{s}}_{1}\right)}\right] .
$$

In what follows, we assume that the background scattering coefficient $\bar{\mu}_{s}$ and the phase function $A\left(\hat{\mathbf{s}}, \hat{\mathbf{s}}^{\prime}\right)$ are known. The first quantity can be, in principle, selected arbitrarily, but it is more practical to define it to be the value of the scattering coefficient in the regions of the sample which are known to be spatially uniform, or in a homogeneous reference sample. Determination of the phase function is a less trivial matter.
The fundamental assumption of SSOT is that $A\left(\hat{\mathbf{s}}, \hat{\mathbf{s}}^{\prime}\right)$ does not depend on position. This is a good approximation in many practical situations, e.g., when the variations in $\mu_{a}$ and $\mu_{s}$ are due to variations in the concentration of scattering and absorbing particles whose shape, however, is on average unchanged. If this proposition is true, then $A\left(\hat{\mathbf{s}}, \hat{\mathbf{s}}^{\prime}\right)$ can be measured experimentally by varying the angle between the source and the detector in such a way that the ray turning point $\mathbf{R}_{21}$ is fixed.

As was mentioned above, the measured intensity in the single-scattering approximation is nonzero only if the vectors $\hat{\mathbf{s}}_{1}$ and $\hat{\mathbf{s}}_{2}$ lie in the same plane. This plane is referred to as a slice and image reconstruction of different slices can be carried out independently. For example, in Fig. 1, the slice coincides with the $y z$-plane of the laboratory frame. To carry out three-dimensional optical imaging, the medium can be approximated by a set of parallel slices, say, $x=n h$, where $n$ is an integer and $h$ is the interslice separation. Inside each slice, $\mu_{t}$ and $\mu_{s}$ can be regarded as functions of the two variables $y$ and $z$. On the other hand, the data is fourdimensional, depending upon two positions and two directions. Indeed, the source and the detector can be scanned independently along the direction of the $Y$ axis. Additionally, the source and detector orientations can be varied. This provides four mathematically independent parameters. The only two restrictions on these parameters are that the ray turning point be inside the medium and that the source and the detector are never aligned on the same axis. Thus, by utilizing multiple incident beams and detecting light exiting the medium at different points and by varying the incidence and exit angles, it is possible to collect sufficient data to reconstruct the absorption and scattering coefficients in a given slice.

Moreover, simultaneous reconstruction of scattering and absorption can be realized without making use of the entire parameter space. It is sufficient to use one angle of incidence and two different angles of detection. This measurement scheme is schematically illustrated in Fig. 2 for the special case of normal incidence and two measurement angles equal to $\pm \pi / 4$. This particular measurement scheme will be now described in more detail.

Consider a beam normally incident on the sample at the position $\left(y_{1}, 0\right)$. Here, all coordinates are in the $y z$ plane. Suppose that measurements are made by a pair of detectors the first of which has the position $\left(y_{2}, L\right)$ and angle $\beta_{2}$ $=\pi / 4$, and the second has position $\left(2 y_{1}-y_{2}, L\right)$ and angle $\beta_{2}=-\pi / 4$, where the angles $\beta_{1}$ and $\beta_{2}$ are defined in Fig. 1 . The case of normal incidence corresponds to $\beta_{1}=0$ and we adopt here a convention according to which the angle $\beta_{2}$ can be either positive or negative, depending on whether the beam exits the medium above or below the normal. We require that $0<y_{2}-y_{1}<L$. The two detectors register photons (rays) that are single-scattered at the same ray turning point (within the sample) $\mathbf{R}_{21}=\left(y_{1}, z_{0}\right)$, where $z_{0}=L-\left(y_{2}-y_{1}\right)$. Such ray pairs are shown in Fig. 2. If we write an integral equation of the form (2) for each of the detectors and subtract one of them from the other, we obtain the following result: 


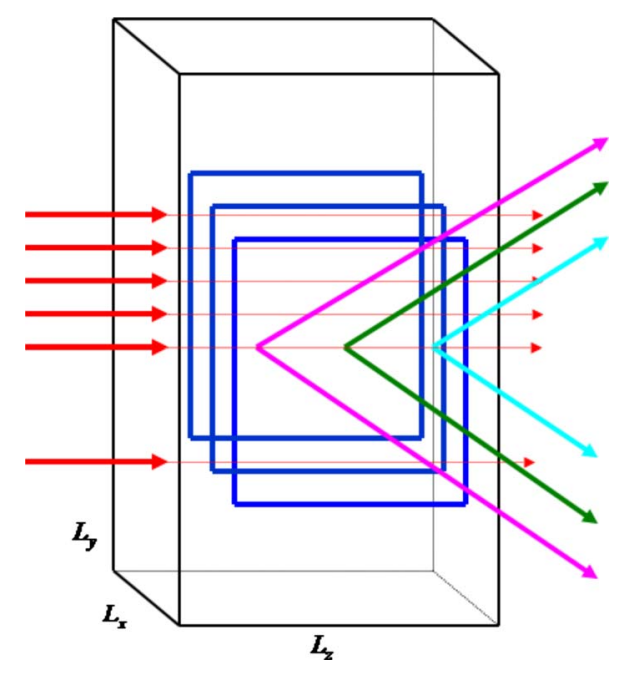

FIG. 2. (Color online) The proposed experiment geometry for simultaneous reconstruction of scattering and absorption coefficients. The rectangles represent the slices in which reconstruction is performed.

$$
\begin{aligned}
\phi\left(y_{2}, \pi / 4 ; y_{1}, 0\right)-\phi\left(2 y_{1}-y_{2},-\pi / 4 ; y_{1}, 0\right) \\
=\int_{\mathrm{BR}\left(y_{2}, \pi / 4 ; y_{1}, 0\right)} \mu_{t}[y(\ell), z(\ell)] d \ell \\
\quad-\int_{\mathrm{BR}\left(2 y_{1}-y_{2},-\pi / 4 ; y_{1}, 0\right)} \mu_{t}[y(\ell), z(\ell)] d \ell .
\end{aligned}
$$

Here, the integrals of the total attenuation coefficient are along the two broken rays described above. Equations (2) and (4) can be solved simultaneously to recover $\mu_{t}(y, z)$ and $\mu_{s}(y, z)$. The absorption coefficient is then obtained as $\mu_{a}$ $=\mu_{t}-\mu_{s}$.

\section{NUMERICAL METHODS}

In Sec. IV below, we illustrate simultaneous reconstruction of scattering and absorption in SSOT based on algebraic inversion of Eqs. (2) and (4). We note that a more sophisticated reconstruction procedure based on an inverse formula is also possible and will be presented elsewhere.

The forward scattering data is obtained for isotropic scattering (that is, for $\left.A\left(\hat{\mathbf{s}}_{2}, \hat{\mathbf{s}}_{1}\right)=1 / 4 \pi\right)$. Note that this choice corresponds to the strong-scattering regime and is, therefore, the most stringent test of the image reconstruction procedure that is based on the single-scattering approximation. The medium containing predetermined inhomogeneities (targets) is discretized on a cubic grid with the step size $h$ which is also equal to the interslice separation used in image reconstruction. The RTE is then solved numerically by the method described in detail in Ref. [8]. Note that, unlike in Ref. [8], here, we have accounted for the spatial dependence of the scattering coefficient. To model noise in the measured data, the specific intensity that was computed numerically was scaled and rounded off so that it was represented by 16-bit unsigned integers, similar to the digital output of a chargecoupled device (CCD) camera. Then, a statistically independent positive-valued random number was added to each measurement. The random numbers were uniformly distributed in the interval $\left[0, n I_{\mathrm{av}}\right]$, where $n$ is the noise level and $I_{\mathrm{av}}$ is the average measured intensity (a 16-bit integer).

The data function is calculated using a discretized version of Eq. (3) [8]. Reconstruction of the attenuation coefficient is obtained using Eqs. (2) and (4). The discrete analog of Eq. (4) is

$$
\sum_{n} \mathcal{L}_{\nu n} \mu_{t n}=\phi_{\nu}
$$

Here, the composite index $\nu=\left(y_{1}, y_{2}\right)$ defines a pair of rays with the same turning point and $\mathcal{L}=\mathcal{L}^{(1)}-\mathcal{L}^{(2)}$, where the matrix elements $\mathcal{L}_{\nu n}^{(1)}$ are given by the length of intersection of the first ray (the one with $\beta_{2}=\pi / 4$ ) with the $n$-th cubic cell of the discretized medium and $\mathcal{L}_{\nu n}^{(2)}$ is the analogous quantity for the second ray (the one with $\beta_{2}=-\pi / 4$ ). The quantity $\mu_{t n}$ is the value of the attenuation coefficient in the $n$-th cubic cell. Finally, $\phi_{\nu}=\phi_{\nu}^{(1)}-\phi_{\nu}^{(2)}$, where $\phi_{\nu}^{(1)}$ and $\phi_{\nu}^{(1)}$ (a) Model
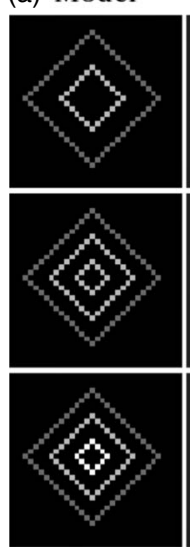

$n=0$
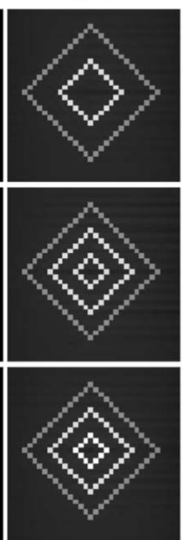

$n=1 \%$
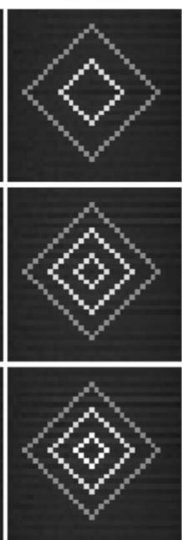

$n=3 \%$
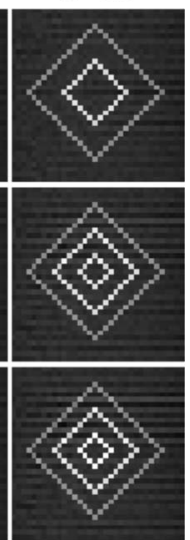

(b) Model
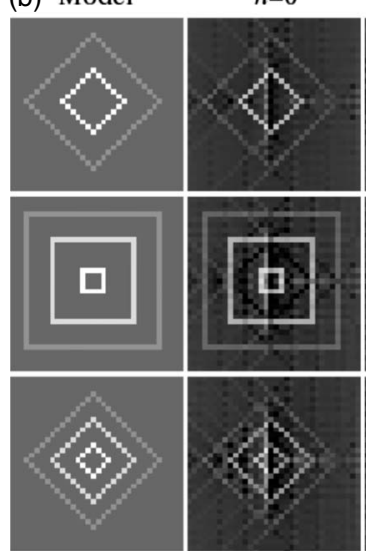
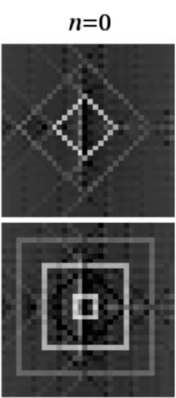

$n=1 \%$
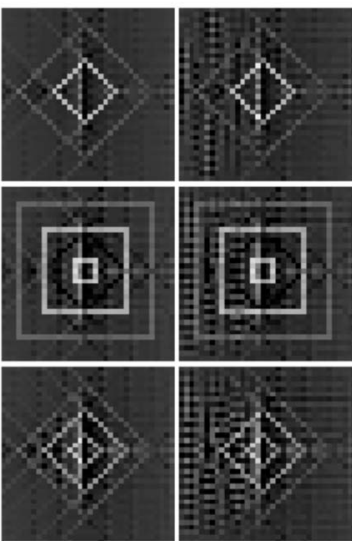

$n=3 \%$
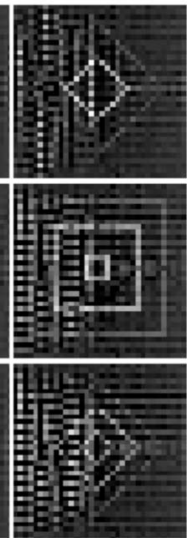

FIG. 3. Reconstruction of the scattering (left) and absorption (right) coefficients for the sample A for various noise levels $n$. The rows in each panel correspond to the slices $x=6 h, 13 h$, and $20 h$ where the inhomogeneities are placed. Scattering and absorbing inhomogeneities are present in the sample simultaneously, and in the slices $x=6$ and $x=20$, they overlap. The top and bottom panels use different color scales (since they visualize different quantities) but all plots within the same panel utilize the same color scale. 
(a) Model
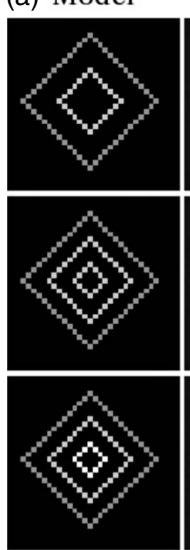

$n=0$
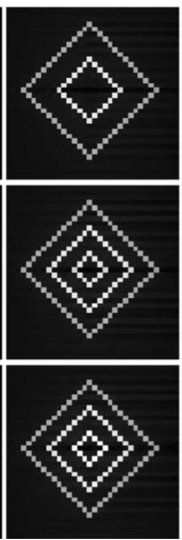

$n=1 \%$
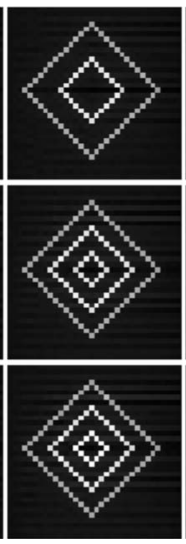

$n=3 \%$
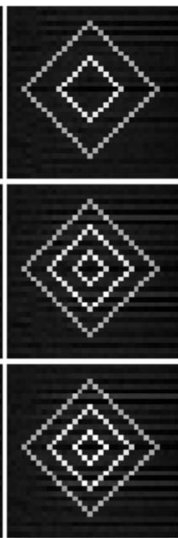

(b) Model

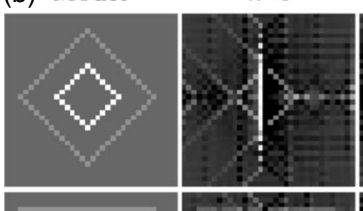

$n=1 \%$

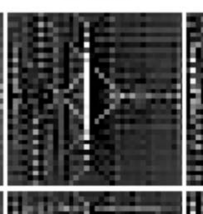

$n=3 \%$

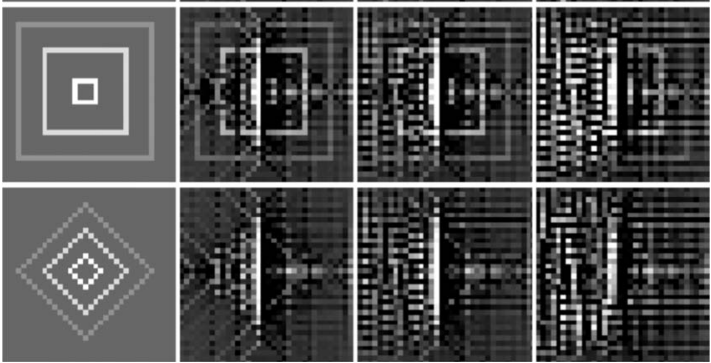

FIG. 4. Same as in Fig. 3 but for the sample B.

are the data points corresponding to the first and second rays, respectively. Equation (5) is solved for $\mu_{t n}$ using a regularized pseudoinverse [9], namely,

$$
\left|\mu_{t}\right\rangle=\left(\mathcal{L}^{*} \mathcal{L}\right)^{-1} \mathcal{L}^{*}|\phi\rangle,
$$

where

$$
\left(\mathcal{L}^{*} \mathcal{L}\right)^{-1}=\sum_{n} \Theta\left(\sigma_{n}^{2}-\epsilon\right) \frac{\left|f_{n}\right\rangle\left\langle f_{n}\right|}{\sigma_{n}^{2}} .
$$

Here, $\epsilon$ is a small regularization parameter. The quantities $\left|f_{n}\right\rangle$ and $\sigma_{n}$ are the singular vectors and singular values, respectively, of the matrix $\mathcal{L}$, obtained by solving the symmetric eigenproblem $\mathcal{L}^{*} \mathcal{L}\left|g_{n}\right\rangle=\sigma_{n}^{2}\left|g_{n}\right\rangle$.

Once $\mu_{t}$ is computed as described above, $\mu_{s}$ can be determined from Eq. (2), discretized as

$$
\ln \left[\frac{\mu_{s}\left(n_{21}\right)}{\bar{\mu}_{s}}\right]=\sum_{n} \mathcal{L}_{\nu n}^{(1)} \mu_{t n}-\phi\left(y_{2}, \pi / 4 ; y_{1}, 0\right),
$$

where $n_{21}$ is the number of voxel containing the turning point of the ray $\operatorname{BR}\left(y_{2}, \pi / 4 ; y_{1}, 0\right)$. Finally, the absorption coefficient is obtained from $\mu_{a n}=\mu_{t n}-\mu_{s n}$.
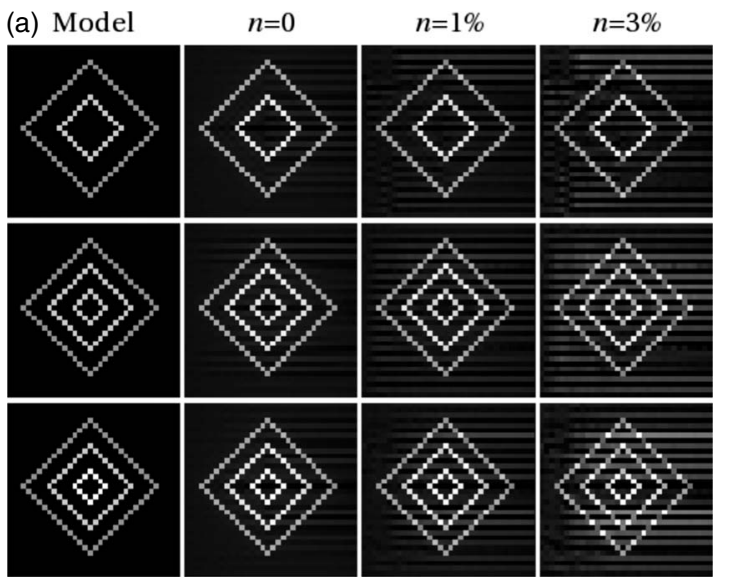

\section{RESULTS}

We have considered four rectangular samples of dimensions $L_{x}=25 h, L_{y}=122 h$, and $L_{z}=40 h$. The absorption and scattering coefficients of the samples were constant everywhere except for a set of voxels where absorbing and scattering inhomogeneities were placed. All such inclusions were present only in the slices $x=6 h, x=13 h$, and $x=20 h$ and are shown by a linear color scale in the columns marked as "Model" in Figs. 3-6 below. In some cases, the absorptive and scattering inhomogeneities overlapped, that is, the same voxel had both its absorption and the scattering coefficients different from the background. The background coefficients $\bar{\mu}_{s}$ and $\bar{\mu}_{a}$, as well as the maximum contrasts of the inclusions (with respect to the background values) are listed in Table I.

The results of numerical reconstruction of the scattering and absorption coefficients for the sample A are shown in Fig. 3. Only the slices containing inhomogeneities are shown in the figure. Intermediate slices have also been reconstructed but appear essentially as black squares and are therefore not shown. As can be seen, good image quality is obtained for the scattering coefficient. The reconstructions are in quantitative agreement with the model and are stable in the presence of noise. The image quality for the absorption

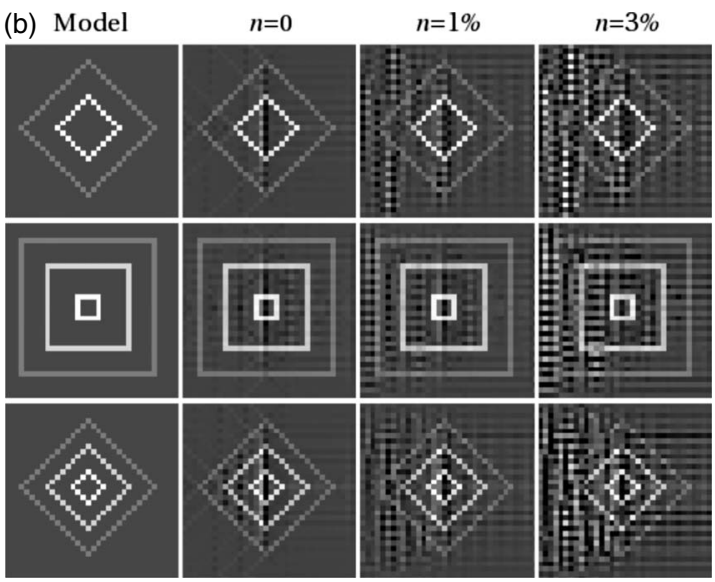

FIG. 5. Same as in Fig. 3 but for Sample C. 
(a) Model
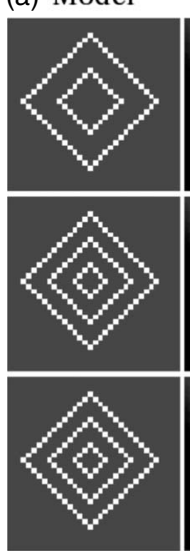

$n=0$
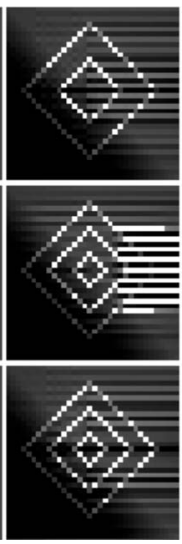

$n=1 \%$

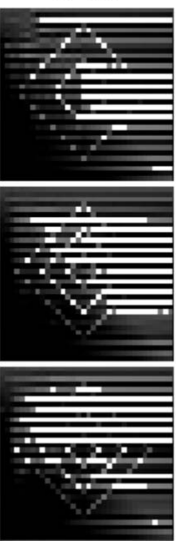

$n=3 \%$

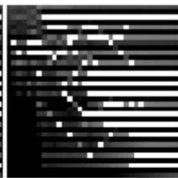

(b) Model

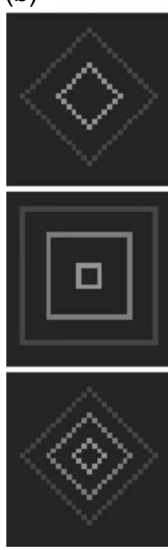

$n=0$
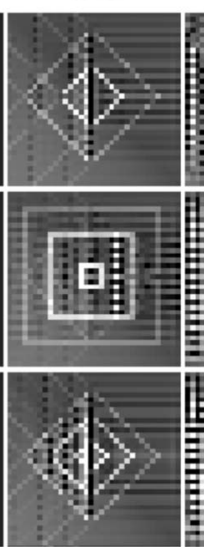

$n=1 \%$

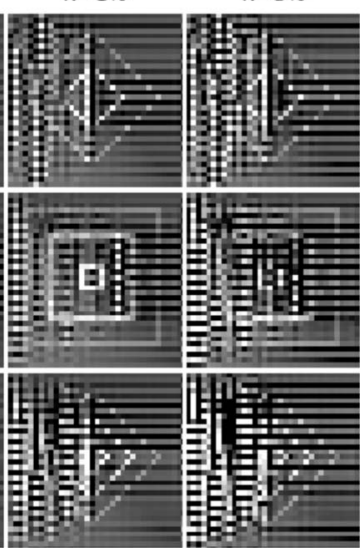

FIG. 6. Same as in Fig. 3 but for Sample D.

coefficient is markedly worse and stronger influenced by noise. However, most of the features of the model can be recognized.

We next consider the sample B in which the strength of all scattering inhomogeneities is increased by a factor of 1.5 , while all other parameters remain the same as in sample A. The results are presented in Fig. 4. Again, a much better image quality is obtained for the scattering than for the absorption coefficient. It can be seen that reconstruction of the absorption coefficient is much more sensitive to noise. This fact is more pronounced in samples with stronger scattering (relative to absorption). This can be explained by noting that $\mu_{a}$ is determined in SSOT by numerically subtracting two relatively large quantities $\left(\mu_{t}\right.$ and $\left.\mu_{s}\right)$ both of which are affected by numerical errors and noise. Consistent with this point, reconstruction of $\mu_{a}$ is more robust when absorbing and scattering inhomogeneities do not overlap, as can be seen from the figures.

To confirm the above conclusion, we have considered sample C, which is strongly absorbing. All parameters of sample $\mathrm{C}$ are the same as in sample $\mathrm{B}$, except that the absorption coefficient was increased uniformly by a factor of 10 (including the absorbing inhomogeneities). The results are shown in Fig. 5. Indeed, it can be seen that image quality for the absorption coefficient in sample $\mathrm{C}$ is markedly better than in samples A and B and is approximately the same as for the scattering coefficient.

Finally, we have considered sample D whose ratio of $\bar{\mu}_{a} / \bar{\mu}_{s}$ is the same as in sample $\mathrm{C}$ (that is, equal to 1 ) but the

TABLE I. Optical properties of the samples used in numerical simulations. The quantities shown are the ratio of the background absorption and scattering coefficients, the optical depth, and the minimum and maximum contrasts of the scattering and absorption coefficients in the inclusions relative to the respective values in the background.

\begin{tabular}{lcccc}
\hline \hline Sample & $\bar{\mu}_{a} / \bar{\mu}_{s}$ & $L_{z} \mu_{s}$ & $\mu_{s} / \bar{\mu}_{s}, \min / \max$ & $\mu_{a} / \bar{\mu}_{a}, \min / \max$ \\
\hline A & 0.1 & 1.6 & $1.33 / 2$ & $2 / 5$ \\
B & 0.1 & 1.6 & $2 / 3$ & $2 / 5$ \\
C & 1.0 & 1.6 & $2 / 3$ & $2 / 5$ \\
D & 1.0 & 3.2 & $2 / 3$ & $2 / 5$ \\
\hline \hline
\end{tabular}

optical depth is twice larger, namely, $\bar{\mu}_{s} L_{z}=3.2$. This is a borderline case where scattering is sufficiently strong so that the single-scattering approximation may be expected to break down. The results of image reconstruction for this sample are shown in Fig. 6. It is remarkable that even when the optical depth is significantly larger than unity, some features in the model remain visible in the reconstruction. Note that for the noise level $n=3 \%$, reconstruction of the scattering coefficient is almost entirely blurred while the reconstruction of the absorption coefficient still resembles the model. Thus, in samples with comparable scattering and absorption but relatively large optical depth, reconstruction of the absorption coefficient is more robust at high levels of noise.

\section{CONCLUSIONS}

We have demonstrated that single-scattering optical tomography allows for simultaneous reconstruction of the scattering and absorption coefficients of mesoscopic systems. We have found that in systems where scattering dominates absorption, quantitative imagine reconstruction of scattering is possible while reconstruction of absorption is much less robust. Simultaneous reconstruction of both coefficients with the same quality can be realized under the condition that scattering and absorption have comparable strengths. This can be experimentally achieved by appropriately choosing the wavelength of the illuminating beam, such that the effects of absorption and scattering have comparable strengths [10]. Further, if the scattering and absorption in the sample are, in fact, of comparable strength, we have found the following: reconstruction of the two coefficients is approximately of the same quality in samples whose optical depth is $\lesssim 1$. In samples of larger optical depth, such as the one considered in Fig. 6, reconstruction of the absorption coefficient is more robust at the levels of noise $n \gtrsim 1 \%$. Reconstruction of samples with optical depth larger than 6 (reconstructions for this optical depth have been demonstrated in Ref. [8]) is not possible since multiple scattering in such systems is sufficiently strong to render the single-scattering approximation inapplicable.

We note that the use of point measurements is not essential for the experimental realization of SSOT. A wide-field 
instrument could, in principle, be constructed wherein a lenscoupled CCD camera is used as a detector and a scanned beam is employed for illumination. Such an arrangement is reciprocal to the experiment proposed in Fig. 1 and would require that the illuminating beam is oriented at a fixed angle with respect to the plane of detection. The primary advantage of this approach is that it would result in significantly reduced data acquisition time.

Finally, we note that the distinct feature of SSOT is that it takes advantage of the salutary effects of scattering. This allows for an increase in the dimensionality of the parameter space on which the data function depends and thereby per- mits the simultaneous reconstruction of two coefficients in the RTE. By comparison, such simultaneous reconstruction is not possible in conventional Radon transform-based imaging modalities such as X-ray computed tomography.

\section{ACKNOWLEDGMENTS}

We thank a referee for a useful suggestion on the experimental feasibility of wide-field SSOT. This work was supported by the NSF under Grants No. DMS-0554100 and No. EEC-0615857, and by the NIH under Grant No. R01EB004832.
[1] C. Vinegoni, C. Pitsouli, D. Razansky, N. Perrimon, and V. Ntziachristos, Nat. Methods 5, 45 (2008).

[2] M. C. W. van Rossum and Th. M. Nieuwenhuizen, Rev. Mod. Phys. 71, 313 (1999).

[3] T. Wilson and C. J. R. Sheppard, Theory and Practice of Scanning Optical Microscopy (Academic Press, New York, 1984).

[4] J. A. Izatt, M. R. Hee, G. M. Owen, E. A. Swanson, and J. G. Fujimoto, Opt. Lett. 19, 590 (1994).

[5] J. Sharpe, U. Ahlgren, P. Perry, B. Hill, A. Ross, J. HecksherSorensen, R. Baldock, and D. Davidson, Science 296, 541 (2002).
[6] T. S. Ralston, D. L. Marks, P. S. Carney, and S. A. Boppart, Nat. Phys. 3, 129 (2007).

[7] S. Arridge, Inverse Probl. 15, R41 (1999).

[8] L. Florescu, J. C. Schotland, and V. A. Markel, Phys. Rev. E 79, 036607 (2009).

[9] F. Natterer and F. Wubbeling, Mathematical Methods in Image Reconstruction (SIAM, Philadelphia, 2001).

[10] A. J. Welch and M. J. C. van Gemert, Optical-Thermal Response of Laser-Irradiated Tissue (Plenum Press, New York, 1995). 